\title{
A COMPARISON OF THE RESPONSE OF PADC NEUTRON DOSEMETERS IN HIGH-ENERGY NEUTRON FIELDS
}

\author{
F. Trompier ${ }^{1, *}$, M. Boschung 2 , A. Buffler ${ }^{3}$, C. Domingo 4 , E. Cale ${ }^{5}$, M.-A. Chevallier ${ }^{1}$, A. Esposito 6 , \\ M. Ferrarini ${ }^{7}$, D. R. Geduld ${ }^{3,8}$, L. Hager ${ }^{9}$, E. Hohmann ${ }^{2}$, S. Mayer ${ }^{2}$, A. Musso ${ }^{5}$, M. Romero-Esposito ${ }^{4}$, \\ S. Röttger ${ }^{10}$, F. D. Smit ${ }^{8}$, A. Sashala Naik ${ }^{11,12}$, R. Tanner ${ }^{9}$, F. Wissmann ${ }^{10}$ and M. Caresana ${ }^{12}$ \\ ${ }^{1}$ Institute for Radiological Protection and Nuclear Safety, BP 17, Fontenay-aux-Roses F-92262, France \\ ${ }^{2}$ Radiation Metrology Section, Paul Scherrer Institute (PSI), Villigen CH-5232, Switzerland \\ ${ }^{3}$ Department of Physics, University of Cape Town, Rondebosch 7700, South Africa \\ ${ }^{4}$ Universitat Autònoma de Barcelona, Barcelona, Spain \\ ${ }_{5}^{5}$ Institute for Radiological Protection and Nuclear Safety, BP 40035, Le Vésinet F-78116, France \\ ${ }^{6}$ IFNF-LNF, FISMEL, via E. Fermi 40, Frascati 00044, Italy \\ ${ }^{7}$ CNAO, Via Privata Campeggi, Pavia 27100, Italy \\ ${ }^{8}$ iThemba Laboratory for Accelerator Based Sciences, Somerset West 7129, South Africa \\ ${ }^{9}$ Public Health England, CRCE, Chilton, Didcot, Oxon OX11 0RQ, UK \\ ${ }^{10}$ Physikalisch-Technische Bundesanstalt (PTB), Bundesallee 100, Braunschweig 38116, Germany \\ ${ }^{11}$ Dipartimento di Energia, Politecnico di Milano, CESNEF, via Ponzio 34/3, Milano 20133, Italy \\ ${ }^{12}$ Mi.Am srl via De Amicis 5, Fabiano di Rivergaro, PC 29029, Italy \\ *Corresponding author: francois.trompier@irsn.fr
}

\begin{abstract}
Within the framework of the EURADOS Working Group 11, a comparison of passive neutron dosemeters in high-energy neutron fields was organised in 2011. The aim of the exercise was to evaluate the response of poly-allyl-glycol-carbonate neutron dosemeters from various European dosimetry laboratories to high-energy neutron fields. Irradiations were performed at the iThemba LABS facility in South Africa with neutrons having energies up to 66 and $100 \mathrm{MeV}$.
\end{abstract}

\section{INTRODUCTION}

Within the framework of the EURADOS Working Group 11, a comparison of passive neutron dosemeters in high-energy neutron fields was organised in 2011 at the iThemba Laboratory for AcceleratorBased Sciences (iThemba LABS) to evaluate the response of passive neutron dosemeters from various European dosimetry laboratories to different highenergy neutron fields. High-energy neutrons have been a subject of investigation for dosimetry for many years due to human space activities and changes in regulations for individual monitoring of aircrew in recent years. The development of new applications with high-energy particle beams for research or cancer treatment (hadrontherapy) with secondary high-energy neutrons, which is a matter of concern for both exposed workers and patients, has driven the need for adapted passive neutron dosimetry. Passive dosemeters used for routine monitoring such as track-etched poly-allylglycol-carbonate (PADC) detectors can be used for such applications, if properly calibrated. From the experimental data obtained in this work, participants could derive calibration factors for high-energy neutron dosimetry or validate track analysis methods developed for this purpose. The second step of WG11 activities will be to organise dosimetry benchmark tests to evaluate the performances of these neutron dosimetry systems in high-energy neutron fields.

\section{MATERIALS AND METHODS}

\section{Layout of beam delivery system}

A separated-sector cyclotron accelerates protons in the energy range from 25 to $200 \mathrm{MeV}^{(1)}$. The ${ }^{7} \mathrm{Li}(p, n)^{7} \mathrm{Be}$ reaction is employed to produce neutrons up to 200 $\mathrm{MeV}$. A 2-m-thick steel collimator with openings at $0^{\circ}$ and $16^{\circ}$ acts as a collimator resulting in $10 \times 10 \mathrm{~cm}^{2}$ shape squared beams at a distance of $8 \mathrm{~m}$ from the target.

\section{Methods for beam characterisation}

The characterisation of the spectral fluence distribution was performed by combining data from a NE213 scintillator and parallel-plate ${ }^{238} \mathrm{U}$ fission chamber ${ }^{(1)}$. The relative neutron energy spectra were determined using the NE213 detector via time-of-flight measurements, and the fluence using the fission chamber. These reference detectors were calibrated by PhysikalischTechnische Bundesanstalt (PTB) in terms of neutron energy, energy threshold and efficiency. 


\section{Beam characteristics}

As shown for the 100-MeV configuration in Figure 1, the neutron spectral fluence distribution at $0^{\circ}$ consists of a high-energy peak and a continuum for lower neutron energy. At $16^{\circ}$, the continuum is similar but the $100-\mathrm{MeV}$ peak is much reduced.

The transition to the ground state and first excited state of ${ }^{7} \mathrm{Be}$ produces quasi-monoenergetic neutron emission. In addition, break-up reactions in lithium cause a low-energy tail below the monoenergetic peak. Reactions $(p, x n)$ with the higher $\mathrm{Z}$ nuclei from the target holder also generate neutrons of lower energies.

\section{PADC description and chemical procedure}

Table 1 summarises the type of PADC used by each participant, as well as the associated procedure used for the etching step. Pre-etching with a mixture of sodium hydroxide and methanol prior to irradiation was performed only by IRSN.

\section{Track analysis}

The track analyses were performed using different approaches and software. Paul Scherrer Institute (PSI) used the full system provided by TASL (microscope and software analysis) ${ }^{(2)}$. This system analyses and characterises each individual track based on 31 different track parameters in order to discriminate etched tracks from background features. At Polimi, the PADC dosemeters were analysed using a commercial reader called Politrack ${ }^{(3)}$, developed at Polimi and marketed by MiAms.r.l. (Italy). Politrack permits two kinds of analysis to be performed: a simple one that just consists of measuring the track density (TD) and a second one based on estimation of the average LET of each track. UAB and IRSN used home developed

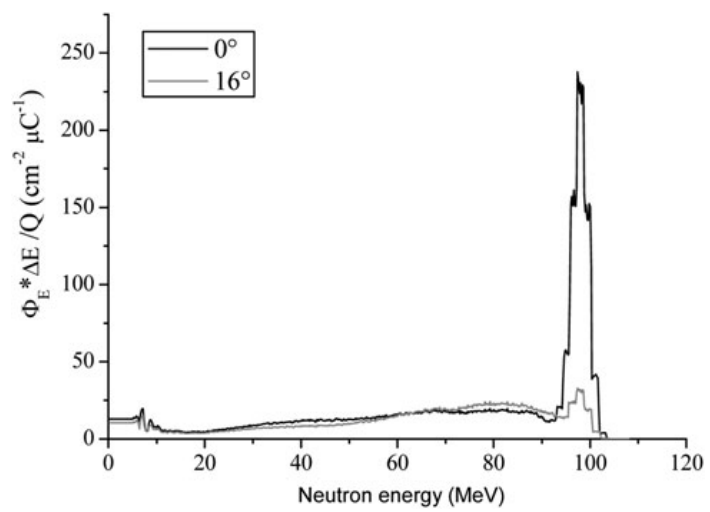

Figure 1. The measured neutron fluence distribution at $0^{\circ}$ and $16^{\circ}$ for $100 \mathrm{MeV}$ protons.
Table 1. Characteristics of PADC and chemical procedures.

\begin{tabular}{|c|c|c|}
\hline Laboratory & $\begin{array}{l}\text { Supplier }(\text { dimension } \\
\left.\qquad\left(\mathrm{mm}^{3}\right)\right)\end{array}$ & Chemical procedure \\
\hline PSI & $\begin{array}{l}\text { TASL }^{\mathrm{a}} \\
(20 \times 25 \times 1.5)\end{array}$ & $\begin{array}{l}6.25 \mathrm{~N} \mathrm{NaOH} \text { for } 2.5 \mathrm{~h} \\
\text { at } 80^{\circ} \mathrm{C}\end{array}$ \\
\hline PHE & $\begin{array}{l}\text { Instrument Plastics } \\
\text { Ltd }(27 \times 39 \times 0.5)\end{array}$ & $\begin{array}{l}5 \mathrm{~N} \mathrm{NaOH} \text { for } 11.5 \mathrm{~h} \text { at } \\
40^{\circ} \mathrm{C}+8 \mathrm{~h} \text { with an } \\
\text { electric field of } 23.5 \mathrm{kV} \\
\mathrm{cm}^{-1}\end{array}$ \\
\hline Polimi & $\begin{array}{l}\text { Intercast }^{\mathrm{b}} \\
(25 \times 25 \times 1.5)\end{array}$ & $\begin{array}{l}6.25 \mathrm{~N} \mathrm{NaOH} \text { for } 1.5 \mathrm{~h} \\
\text { at } 98^{\circ} \mathrm{C}\end{array}$ \\
\hline UAB & $\begin{array}{l}\text { Intercast } \\
(20 \times 20 \times 1)\end{array}$ & $\begin{array}{l}3 \text {-step ECE }: 20 \mathrm{kV} \\
\mathrm{cm}^{-1} \text { at } 50 \mathrm{kHz} \text { for } 5 \\
\mathrm{~h}+2 \mathrm{kHz} \text { for } 1 \mathrm{~h}+15 \text { - } \\
\text { min chemical post- } \\
\text { etching }\end{array}$ \\
\hline IRSN & $\begin{array}{l}\text { TASL }^{\mathrm{a}} \\
\text { Technol }^{\mathrm{d}} \\
(20 \times 25 \times 1.5)\end{array}$ & $\begin{array}{l}6.25 \mathrm{~N} \mathrm{NaOH} \text { for } 15 \mathrm{~h} \\
\text { at } 70^{\circ} \mathrm{C}\end{array}$ \\
\hline
\end{tabular}

\footnotetext{
aTrack Analysis System Ltd, Bristol.

${ }^{\mathrm{b}}$ Intercast Europe S.r.1., Parma.

${ }^{\mathrm{c}}$ Electro-chemical etching.

${ }^{\mathrm{d}}$ Chiyoda Technol Corporation.
}

systems to count the tracks. UAB imposed a threshold on track size to discriminate etched tracks from background. The routine procedure with such discrimination was not used for IRSN to analyse the raw data. PHE used a system developed for them, which has a minimum track size and applies corrections to eliminate irregular tracks and for linearity ${ }^{(4)}$. Because UAB and PHE use electrochemical etch systems, the tracks are large and easily counted. The other three systems have smaller tracks of different sizes, so the resolution and track size threshold of the read system will be a critical factor.

\section{Experimental set-up}

The dosemeters were irradiated at 8 metres from the target. Irradiations were performed at emission angles of $0^{\circ}$ and $16^{\circ}$ simultaneously. For a given configuration, a minimum of four dosemeters were irradiated. For the Polimi PADC, $1 \mathrm{~cm}$ of PMMA was used in front of the PADC as a converter and to ensure the full charged particle equilibrium. The UAB system used 3-mm polyethylene $+300-\mu \mathrm{m}$ Makrofol $^{\circledR}+100-\mu \mathrm{m}$ Nylon ${ }^{\circledR}$ as a converter, and a 5 -mm lead sheet was added to the dosemeter housing to investigate the increase of sensitivity expected for high-energy neutrons. PADC from PSI, PHE and IRSN were irradiated in the personal dosemeter holder use routinely. Irradiations were performed in air or on slab PMMA phantom. The integrated doses on PADC ranged between 2 and 7 $\mathrm{mSv}$ in terms of $H_{\mathrm{p}}(10)$ or $H^{*}(10)$, depending on irradiation configurations (air or phantom). It should be 


\section{F. TROMPIER ET AL.}

noted that dosemeters that did not use additional material in front of the detector may have a component of response from the charged particles generated in air.

\section{Reference dosimetry}

For each irradiation, the fluence was determined from monitors calibrated by PTB. For each irradiation configuration, a conversion factor from fluence to operational quantities was derived from the combination of measured neutron spectra and the monoenergetic conversion factors from ICRU report $57^{(5)}$. For $H_{\mathrm{p}}(10)$ of $>20 \mathrm{MeV}$, as no conversion factor is available in ICRU57, conversion factors for $H^{*}(10)$ were used: this should be acceptable because the irradiations were for normal incidence and $H^{*}(10)$ and $H_{\mathrm{p}}\left(10,0^{\circ}\right)$ then only differ because of the shape of the phantom. Table 2 summarises the mean neutron energy and the conversion coefficients for each irradiation configuration. The total uncertainty on the fluence measurements has been estimated at $\sim 10 \%$.

\section{RESULTS}

\section{Irradiation on phantom}

The results of the irradiations performed on phantom for the four configurations are presented in Figure 2. Each point represents the average of the TD per $\mathrm{mSv}$ for the different detectors exposed in that configuration. The uncertainty is the combination of the standard deviation on TD and the total uncertainty on reference dose. The TD reported by PSI is found to be on average about a factor of 2.6 lower than the 4 other PADC types. The 4 other types have close TD ranging between 120 and 160 tracks $\mathrm{cm}^{-2} \mathrm{mSv}^{-1}$. The difference between IRSN (TASL) and UAB is $\sim 10 \%$ and $\sim 6 \%$ between UAB and PHE.

Whilst the readings on the IRSN (TASL) and PSI dosemeters are very different, they do exhibit similar behaviour: the variation for the three configurations with the lowest mean neutron energies is lower than uncertainties whereas a slight decrease is observed for the highest mean energy configuration (15 and $28 \%$ for IRSN-TASL and PSI). Although IRSN and PSI

Table 2. Ambient and personal dose equivalents per unit neutron fluence delivered for each irradiation configuration.

\begin{tabular}{lccc}
\hline Configuration & $\begin{array}{c}E_{\text {mean }} \\
(\mathrm{MeV})\end{array}$ & $\begin{array}{c}H^{*}(10) \text { per } \\
\text { unit fluence } \\
(\mathrm{pSv} \mathrm{cm})\end{array}$ & $\begin{array}{c}H_{\mathrm{p}}(10) \text { per } \\
\text { unit fluence } \\
\left.(\mathrm{pSv} \mathrm{cm})^{2}\right)\end{array}$ \\
\hline $100 \mathrm{MeV}, 0^{\circ}$ & 68.1 & 342.2 & 344.1 \\
$100 \mathrm{MeV}, 16^{\circ}$ & 58.8 & 363.3 & 366.1 \\
$66 \mathrm{MeV}, 0^{\circ}$ & 44.2 & 413.5 & 416.9 \\
$66 \mathrm{MeV}, 16^{\circ}$ & 37.4 & 439.2 & 443.7 \\
\hline
\end{tabular}

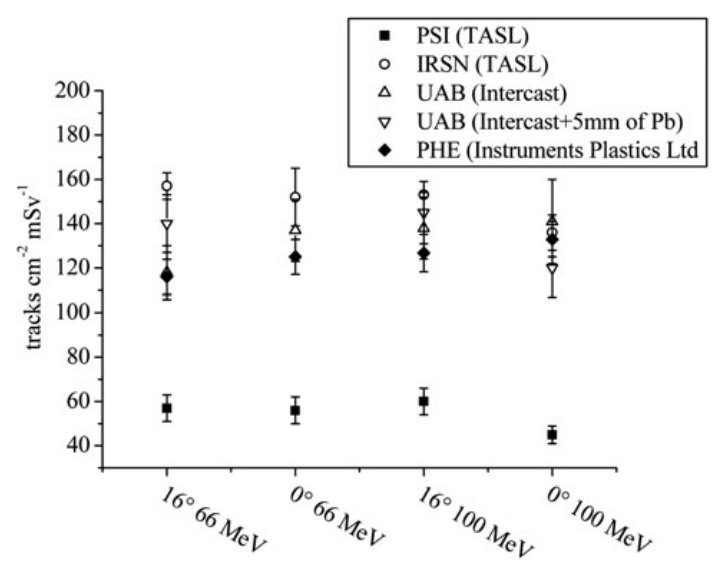

Figure 2. A comparison of TD per $\mathrm{mSv}$ for PADC irradiated on phantom for four different configurations.

have used the same PADC, i.e. both TASL, it is difficult to identify a specific cause for the observed difference in TD values. The etching procedures, the track analysis methods and the housings of the dosemeter are different and may have some influence. For the $\mathrm{UAB}$ and PHE detectors, the variations observed between the configurations are of the same magnitude as the uncertainties. For UAB PADC, the addition of $5 \mathrm{~mm}$ of lead does not lead to an increase in the sensitivity. Obviously, a thicker layer might be effective but that can hardly be envisaged for a personal dosemeter.

\section{Irradiation in air}

The results of the irradiation performed in air for the four configurations are presented on Figure 3. As for irradiation on phantom, UAB and IRSN (TASL) PADC have a similar TD. The difference between the air and phantom configurations is of the same magnitude as the uncertainties. IRSN PADC (TASL) shows a similar behaviour as in air, with a slight decrease for the highest mean energy. Technol PADC is slightly more sensitive than the TASL ones by $\sim 10 \%$. But these results are hardly interpretable as the procedure is not optimised at these levels of energy and no specific procedure adapted to the type of PADC was developed. For UAB PADC, the $5 \mathrm{~mm}$ of lead in front of the PADC has no influence on the TD, as for irradiation on phantom. The TD of Polimi PADC is about twice as high as those from other PADC. If Polimi and UAB have used the same PADC, it is difficult to explain the observed difference, as the etching procedure, the track analysis method and the housing of the dosemeter are different and may have some influence. 


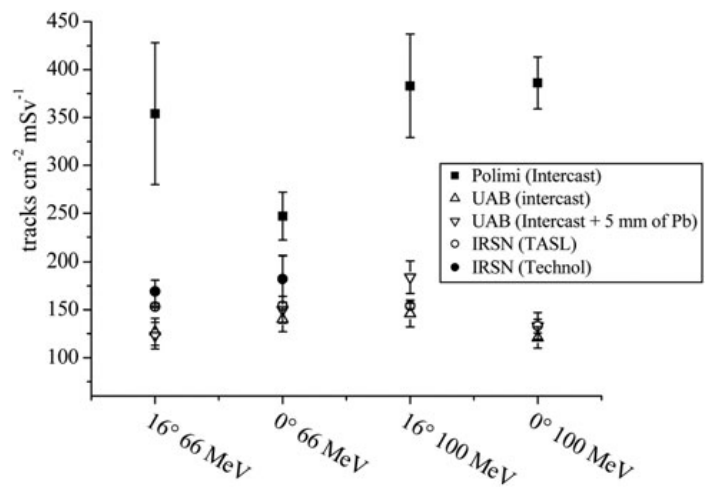

Figure 3. A comparison of $\mathrm{TD}$ per $\mathrm{mSv}$ for PADC irradiated in air for four different configurations.

For Polimi, except for the $0^{\circ}$ and $66-\mathrm{MeV}$ configuration, the TD variation is within the uncertainties. For the $0^{\circ}$ and $66-\mathrm{MeV}$ configuration, the TD decreases by a factor of 1.5 relatively to other configurations. Nevertheless, it is worth noting that the Politrack analysis software is designed to estimate dose through LET estimation of each track; the track counting mode not being used routinely ${ }^{(6)}$. In Figure 4, the relative TD and dose sensitivity are compared. The decrease for the $0^{\circ}$ and $66-\mathrm{MeV}$ configuration is not observed anymore in the dose mode.

\section{CONCLUSION}

For the different neutron spectra investigated in this work, the TD of each PADC type varies only weakly, compared with the energy dependence observed at lower energy $(<20 \mathrm{MeV})$. For the PADC presenting the lowest uncertainties, a slight decrease of the TD has been observed for the highest mean energy (68.1 $\mathrm{MeV})$. The difference of TD observed between the different detectors, which reaches a factor of 6 between the minimum and maximal values, cannot be explained without further investigations, but it is likely to be linked to track size: the ratio of the track diameters for PSI, IRSN and Polimi should be $\sim$ 1:3:6 based on etch duration and temperature. With these experiments, participants had the possibility of calibrating their detectors to high-energy neutrons or to validate specific methods for dose estimation. Based on these data, the dosimetry performances of PADC of participants will be evaluated in future benchmarking experiments.

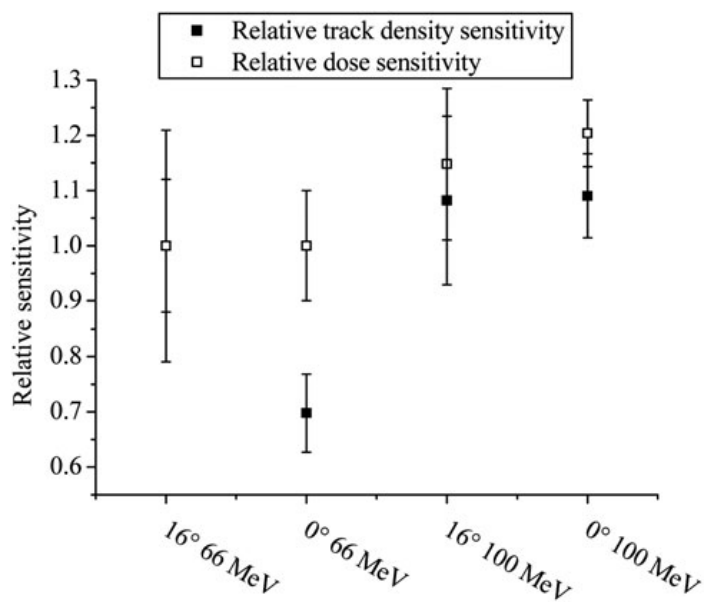

Figure 4. A comparison of relative sensitivity in terms of TD and $H^{*}(10)$ for POLIMI PADC irradiated in air for four different configurations.

\section{ACKNOWLEDGEMENTS}

The authors thank all the staff from iThemba LABS for their kind help during the beam time.

\section{REFERENCES}

1. Mosconi, M., Musonza, E., Buffler, A., Nolte, R., Röttger, S. and Smit, F. D. Characterisation of the highenergy neutron beam at iThemba LABS. Radiat. Meas. 45, 1342-1345 (2010).

2. Mayer, S., Assemnbacher, F. and Boschung, M. Determination of the response function for two personal neutron dosemeter designs based on PADC. Radiat. Prot. Dosim., submitted in the proceeding of Neudos 12 conference.

3. Caresana, M., Ferrarini, M., Fuerstner, M. and Mayer, S. Determination of LET in PADC detectors through the measurement of track parameters. Nucl. Instrum. Meth. A 683, 8-15 (2012).

4. Tanner, R. J., Hager, L. G. and Bartlett, D. T. Improved characterisation of the HPA PADC neutron personal dosemeter Radiat. Prot. Dosim. 125, 254-257 (2007).

5. International Commission on Radiation Units and Measurements. Conversion coefficients for use in radiological protection against external radiation, ICRU report $n^{\circ}$ 57. ISBN 0-913394-56-4 (1998).

6. Caresana, M., Ferrarini, M., Parravicini, A. and Sashala Naik, A. Evaluation of a personal and environmental dosimeter based on cr-39 track detectors in quasi-monoenergetic neutron fields. Radiat. Prot. Dosim., submitted in the proceeding of Neudos 12 conference. 